\title{
Some 'homological' properties of the stable Higson corona
}

\author{
Rufus Willett
}

\begin{abstract}
We establish certain 'homological properties' of the stable Higson corona used by Emerson and Meyer to study the Dirac-dual-Dirac approach to the Baum-Connes conjecture [5]. These are used to obtain explicit isomorphisms between the K-theory groups of stable Higson coronas, and the K-theory groups of certain geometrically defined boundaries. This is sufficient to give a simple proof of the strong Novikov conjecture for torsion-free hyperbolic groups and torsion-free groups acting properly and cocompactly on CAT(0) spaces, and also provides an input into an index theorem in single operator theory [15], [16].
\end{abstract}

Mathematics Subject Classification (2010). 46L80.

Keywords. K-theory, Baum-Connes conjecture, coarse geometry.

\section{Introduction}

Let $X$ be a proper metric space. In work on the Baum-Connes conjecture [5] Emerson and Meyer introduce the stable Higson corona $c(X)$ (see Definition 2.2 below), a 'large-scale' geometric invariant of $X$. They develop some further properties of this object in [4], in particular putting (a reduced version of) it in K-theoretic duality with the Roe algebra of $X, \mathrm{C}^{*}(X)$. Indeed, it follows from [4], Theorem 6.1, that there exists a natural pairing

$$
K_{*}\left(\mathrm{c}^{\mathrm{red}}(X)\right) \otimes K_{*}\left(C^{*}(X)\right) \rightarrow \mathbb{Z}
$$

which is moreover rationally nondegenerate in many interesting cases: for example, if the space $X$ is uniformly contractible, and the coarse assembly and coarse coassembly maps are both isomorphisms.

This suggests a close analogy between the stable Higson corona and the Roe algebra, which we aim to develop in this piece. Specifically, we show that the Ktheory of the stable Higson corona has certain 'homological properties', such as satisfying a Mayer-Vietoris sequence for coarsely excisive [11] decompositions of metric spaces, and invariance under coarse homotopy equivalence [8]. These closely mirror properties of the Roe algebra that were proved in [11], [8], [9], [7] by Higson, Pedersen, Roe and Yu. This approach to the stable Higson corona was suggested to the author by John Roe. 
Our principal motivation was to provide a relatively simple and self-contained proof of an index theorem in single operator theory [16], [15], following on from work of Rabinovich, Roch and Silbermann [13], and Rabinovich, Roch and Roe [12]. The main ingredient necessary for this application to operator theory is the first part of the theorem below; it is a corollary of the homological properties that we establish for the stable Higson corona (see Propositions 4.3 and 4.5 below, and Section 4 for definitions).

Theorem 1.1. Say that $X$ is a proper geodesic CAT(0) metric space, $\partial_{v} X$ its visual boundary, and $\mathcal{K}$ a copy of the compact operators on a separable infinite dimensional Hilbert space. Then there is natural inclusion

$$
i: C\left(\partial_{v} X\right) \otimes \mathcal{K} \rightarrow c(X)
$$

which induces an isomorphism on $K$-theory.

Say that $X$ is a proper quasi-geodesic Gromov hyperbolic space, $\partial_{G} X$ its Gromov boundary and $\mathcal{K}$ a copy of the compact operators on a separable infinite dimensional Hilbert space. Then there is a natural inclusion

$$
i: C\left(\partial_{G} X\right) \otimes \mathcal{K} \rightarrow c(X) .
$$

which induces an isomorphism on K-theory.

It is a simple corollary that the coarse co-assembly map of Emerson and Meyer is an isomorphism; moreover, the strong Novikov conjecture for torsion-free groups acting on such spaces follows from this. This is not a new result, and indeed follows from the work of Emerson and Meyer on the stable Higson corona [4]. Nonetheless, the proofs given here are elementary, in particular, using only K-theory and no bivariant KK-theory; they thus provide a simple approach to the Novikov conjecture for such groups.

Outline of the article. Section 2 below recalls the definition of the stable Higson corona and some of its basic properties from [4]. Section 3 proves our main results on homological properties of the stable Higson corona. Section 4 concludes the piece with some concrete geometric examples: roughly, in cases of non-positive curvature the results of Section 3 can be used to show that the inclusion of the continuous functions on a geometrically defined boundary into the stable Higson corona induces an isomorphism on K-theory; a very special case of such a result is what we need for the index theorem of [16]. The connection with the coarse co-assembly map, and thus with the strong Novikov conjecture, is pointed out at the end of the section. 


\section{Definitions}

Although almost everything below could be done in the context of general coarse spaces (see [15], Chapter 4 and Appendix A), we choose to restrict ourselves to the case of metric spaces. This is mainly to keep the piece more self-contained and readable, but also as all the coarse spaces we are interested in are metrizable. Throughout then, $X, Y, Z$ denote proper metric spaces; 'proper' means here that closed balls are compact. The main examples we have in mind are finitely generated groups equipped with a word metric, and complete Riemannian manifolds.

The following definition fixes the precise frameworks we work in.

Definition 2.1. A function $f: X \rightarrow Y$ is called a coarse map if it is proper, and if for all $R>0$ there exists $S>0$ such that if $d_{X}\left(x, x^{\prime}\right) \leq R$ then $d_{Y}\left(f(x), f\left(x^{\prime}\right)\right) \leq S$. Two coarse maps $f, g: X \rightarrow Y$ are called close if there exists $C \geq 0$ such that $d_{Y}(f(x), g(x)) \leq C$ for all $x \in X$.

The category $\mathcal{C}$ (for 'continuous coarse') has objects proper metric spaces and morphisms continuous coarse maps.

The category $\ell$ (for 'coarse') has objects proper metric spaces and morphisms equivalence classes of coarse maps, where the equivalence relation is defined by closeness.

Our main objects of study are taken from [4], Definitions 3.1, 3.2 and 5.4; for the reader's convenience, we repeat these definitions below.

Definition 2.2. Let $A$ be a normed vector space and $f: X \rightarrow A$ be a bounded map. Then for any $R>0$ the variation of $f$ at scale $R$, a function $\nabla_{R} f: X \rightarrow \mathbb{R}_{+}$, is defined by

$$
\left(\nabla_{R} f\right)(x):=\sup \left\{\|f(x)-f(y)\|_{A} \mid d(x, y) \leq R\right\} .
$$

The map $f$ is said to be of vanishing variation if for all $R>0, \nabla_{R} f$ tends to zero at infinity.

Let now $\mathcal{K}=\mathcal{K}(\mathscr{H})$ denote a copy of the compact operators on a separable infinite dimensional Hilbert space. The stable Higson compactification of $X$ is the $\mathrm{C}^{*}$-algebra

$$
\bar{c}(X):=\{f: X \rightarrow \mathcal{K} \mid f \text { is continuous, bounded and of vanishing variation }\} .
$$

Note that it contains the $\mathrm{C}^{*}$-algebra $C_{0}(X, \mathcal{K})$ of continuous functions from $X$ to $\mathcal{K}$ that vanish at infinity as an ideal. Emerson and Meyer define the stable Higson corona of $X$ to be the $\mathrm{C}^{*}$-algebra

$$
c(X):=\frac{\bar{c}(X)}{C_{0}(X, \mathcal{K})} .
$$


Let now $\mathscr{B}=\mathscr{B}(\mathscr{H})$ denote a copy of the $\mathrm{C}^{*}$-algebra of bounded operators on a separable infinite dimensional Hilbert space $\mathcal{H}$. The reduced stable Higson compactification is defined to be the $\mathrm{C}^{*}$-algebra

$$
\begin{array}{r}
\bar{c}^{\text {red }}(X):=\{f: X \rightarrow \mathscr{B} \mid f \text { is continuous, bounded, of vanishing variation } \\
\text { and satisfies } f(x)-f(y) \in \mathcal{K} \text { for all } x, y \in X\} .
\end{array}
$$

The reduced stable Higson corona is defined to be the quotient

$$
c^{\mathrm{red}}(X):=\frac{\bar{c}^{\mathrm{red}}(X)}{C_{0}(X, \mathcal{K})} .
$$

The following collects together [4], Proposition 3.7, Lemma 3.10 and Proposition 5.5, all of which we will need.

Proposition 2.3. (1) The assignments $X \mapsto \bar{c}(X)$ and $X \mapsto \bar{c}^{\text {red }}(X)$ give rise to contravariant functors from the category $e^{e}$ to the category of $\mathrm{C}^{*}$-algebras and *-homomorphisms; the assignments $X \mapsto c(X)$ and $X \mapsto c^{\text {red }}(X)$ give rise to contravariant functors from the category $\mathcal{C}$ to the category of $\mathrm{C}^{*}$-algebras and *-homomorphisms.

(2) If $X$ is noncompact, then the inclusions $\mathcal{K} \rightarrow \bar{c}(X), c(X)$ as constant functions induce injections on K-theory. Moreover, in this case the natural inclusions $\bar{c}(X) \rightarrow$ $\bar{c}^{\mathrm{red}}(X), \mathrm{c}(X) \rightarrow \mathrm{c}^{\mathrm{red}}(X)$ induce isomorphisms

$$
K_{*}\left(\bar{c}^{\mathrm{red}}(X)\right) \cong \frac{K_{*}(\overline{\mathrm{c}}(X))}{K_{*}(\mathcal{K})} \quad \text { and } \quad K_{*}\left(\mathrm{c}^{\mathrm{red}}(X)\right) \cong \frac{K_{*}(\mathrm{c}(X))}{K_{*}(\mathcal{K})}
$$

Remarks 2.4. (1) In the references cited above Emerson and Meyer show functoriality of $X \mapsto c(X), c^{\text {red }}(X)$ only for closeness classes of Borel coarse maps. However, it follows from our assumptions that any coarse map can be assumed Borel up to closeness (see [15], Remark A.6), so we do get functoriality on the category $\ell$; this minor point makes the details of the arguments in Section 4 slightly easier.

(2) In the case that $X$ is compact (equivalently, bounded), part (2) above fails. Indeed, in this case $c(X)=0$ while $c^{\text {red }}(X)$ is isomorphic to the Calkin algebra; we will use this in Example 3.8 below.

(3) Emerson and Meyer also define a version of the stable Higson corona with coefficients in another $\mathrm{C}^{*}$-algebra. To keep notation uncluttered we will not discuss this explicitly, but it is not hard to see that all of our proofs carry over without change to this more general case.

\section{Homological properties of the stable Higson corona}

For brevity, we restrict attention in the main part of this section to the reduced corona functor from Definition 2.2; some additional results for ${ }^{\text {red }}(X)$ are included at the end of the section. 
From now on, if $\phi: X \rightarrow Y$ is a coarse map, then we denote by

$$
\phi^{\sharp}: c^{\text {red }}(Y) \rightarrow c^{\text {red }}(X) \quad \text { and } \quad \phi^{*}: K_{*}\left(c^{\text {red }}(Y)\right) \rightarrow K_{*}\left(c^{\text {red }}(X)\right)
$$

its image under the functors $c^{\text {red }}(\cdot)$ and $K_{*}\left(c^{\text {red }}(\cdot)\right)$ respectively. We use the same notation for the images of continuous coarse maps under the reduced stable Higson compactification functor and its composition with the K-theory functor, as well as the unreduced versions of all these functors.

As a first step we establish a Mayer-Vietoris type sequence for the functor $X \mapsto$ $K_{*}\left(c^{\text {red }}(X)\right)$; Emerson and Meyer point out that something like this exists ([4], Remark 5.7) but do not develop it any further. For a decomposition $X=A \cup B$ to give rise to such a sequence, it must satisfy some notion of excision appropriate to the coarse category; that used below is taken from [11], Definition 1.1.

Definition 3.1. For any subspace $Y$ of $X$ and $R>0$ define

$$
N(Y, R)=\{x \in X \mid d(x, y)<R \text { for some } y \in Y\} .
$$

Let $X=A \cup B$ be a decomposition of $X$ into closed subspaces. It is called coarsely excisive if for all $R>0$ there exists $S>0$ such that

$$
N(A, R) \cap N(B, R) \subseteq N(A \cap B, S) .
$$

Example 3.2. $\mathbb{R}=(-\infty, 0] \cup[0, \infty)$ is a coarsely excisive decomposition, but

$$
\begin{aligned}
X: & =\left\{(x, y) \in[-\pi / 2, \pi / 2] \times \mathbb{R}_{+}|y=| \tan (x) \mid\right\} \\
& =\{(x, y) \in X \mid x \leq 0\} \cup\{(x, y) \in X \mid x \geq 0\}
\end{aligned}
$$

(equipped with the subspace metric from $\mathbb{R}^{2}$ ) is not.

The next lemma is closely related to [11], Proposition 2.1.

Lemma 3.3. Let $X=A \cup B$ be a decomposition of $X$ into closed subspaces. Let $i_{A}: A \rightarrow X, i_{B}: B \rightarrow X, j_{A}: A \cap B \rightarrow A, j_{B}: A \cap B \rightarrow B$ be the associated inclusion maps.

Then if $X=A \cup B$ is coarsely excisive there is a pullback diagram of $\mathrm{C}^{*}$-algebras

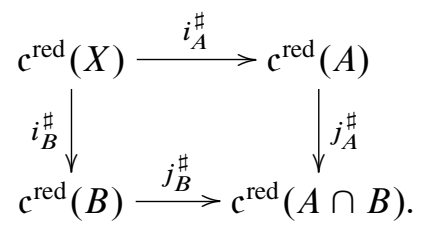


Proof. Pullbacks always exist in the category of $\mathrm{C}^{*}$-algebras and $*$-homomorphisms: the pullback $P$ over the diagram

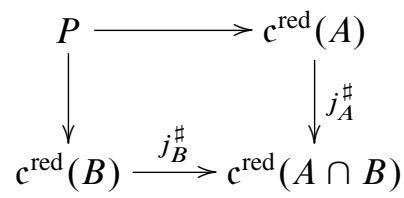

can be concretely constructed as

$$
P=\left\{(a, b) \in c^{\mathrm{red}}(A) \oplus \mathrm{c}^{\mathrm{red}}(B) \mid j_{A}^{\#}(a)=j_{B}^{\sharp}(b)\right\},
$$

equipped with the obvious projection maps to $c^{\text {red }}(A)$ and $c^{\text {red }}(B)$.

If $Y$ is a proper metric space, denote the image of $f \in \overline{\mathrm{c}}^{\mathrm{red}}(Y)$ under the quotient map to $c^{\text {red }}(Y)$ by $[f]$. To show the existence of the diagram (1) above it suffices to prove that the map $c^{\text {red }}(X) \rightarrow P$ defined by

$$
[f] \mapsto\left(\left[\left.f\right|_{A}\right],\left[\left.f\right|_{B}\right]\right)
$$

is an isomorphism of $\mathrm{C}^{*}$-algebras. It is not hard to check that it is a well-defined, injective $*$-homomorphism. Surjectivity is shown below.

Say then that $\left(\left[g_{A}\right],\left[g_{B}\right]\right)$ is an element of $P$. It follows from the definition of $P$ that there exists $h \in C_{0}(A \cap B, \mathcal{K})$ such that $g_{A}-g_{B}=h$ on $A \cap B$. Surjectivity of the restriction map $C_{0}(B, \mathcal{K}) \rightarrow C_{0}(A \cap B, \mathcal{K})$ implies that $h$ extends to an element of $C_{0}(B, \mathcal{K})$, which we also denote $h$. Define a function $f: X \rightarrow \mathcal{K}$ by

$$
f(x)= \begin{cases}g_{A}(x), & x \in A, \\ g_{B}(x)+h(x), & x \in B .\end{cases}
$$

This is well defined as $g_{A}(x)=g_{B}(x)+h(x)$ on $A \cap B$. If $f$ is in $\bar{c}^{\text {red }}(X)$, then $[f] \in \mathfrak{c}^{\text {red }}(X)$ maps to $\left(\left[g_{A}\right],\left[g_{B}\right]\right)$ under the $*$-homomorphism in line (2) above. The function $f$ is continuous and bounded as $g_{A}$ and $g_{B}+h$ are, and as they agree on the closed subset of $X$ where both are defined. Moreover, assuming $A$ and $B$ are non-empty (indeed, if one is empty the lemma is trivial), the coarse excisiveness condition forces $A \cap B$ to be non-empty, whence $f(x)-f(y)$ is compact for all $x, y \in X$. It thus suffices to show that $f$ is of vanishing variation.

To this end, let $R, \varepsilon>0$. Then there exists $S \geq R$ such that

$$
N(A, R) \cap N(B, R) \subseteq N(A \cap B, S) .
$$

Moreover, by vanishing variation of $g_{A}$ on $A$ and $g_{B}+h$ on $B$, there exist compact subsets $K_{A} \subseteq A, K_{B} \subseteq B$ such that for all $a \in A \backslash K_{A}$ (resp. $b \in B \backslash K_{B}$ )

$$
\left|\left(\nabla_{S} g_{A}\right)(a)\right|<\varepsilon \quad\left(\text { resp. }\left|\left(\nabla_{S}\left(g_{B}+h\right)\right)(b)\right|<\varepsilon\right) .
$$


Adopting the convention that if one of $\left(\nabla_{S} f\right)(x),\left(\nabla_{S} g\right)(x)$ does not make sense then we just set it zero, it follows that for all $x \in X \backslash\left(K_{A} \cup K_{B}\right)$,

$$
\left|\left(\nabla_{R} f\right)(x)\right| \leq \max \left\{\left|\left(\nabla_{S} g_{A}\right)(x)\right|,\left|\left(\nabla_{S}\left(g_{B}+h\right)\right)(x)\right|\right\}<\varepsilon .
$$

This shows that $f$ has vanishing variation, completing the proof.

The second ingredient needed to establish a Mayer-Vietoris sequence is surjectivity of the restriction maps $j_{A}^{\#}$ and $j_{B}^{\#}$.

Lemma 3.4. Let $A \subseteq X$ a closed subspace. Then the restriction $*$-homomorphism

$$
\bar{c}^{\mathrm{red}}(X) \rightarrow \bar{c}^{\mathrm{red}}(A)
$$

is surjective.

Unfortunately the proof given below is a little technical; see [3], Theorem 1.4, for a simpler proof in the 'classical' case of complex-valued functions.

Proof. Let $f \in \bar{c}^{\text {red }}(A)$ and $\varepsilon>0$. As $*$-homomorphisms have closed image, it suffices to prove that there exists $\tilde{f} \in \bar{c}^{\text {red }}(X)$ such that $\|\tilde{f}(a)-f(a)\|_{\mathcal{B}(\mathscr{H})}<\varepsilon$ for all $a \in A$.

Fix a basepoint $a_{0} \in A$ and let

$$
v: A \rightarrow \mathbb{R}_{+} \cup\{\infty\}
$$

be any function that is non-decreasing with respect to distance from $a_{0}$, tends to infinity at infinity in $A$, and is such that

- $v(a) \leq \sup \left\{R>0 \mid\left(\nabla_{R} f\right)(a)<\varepsilon\right\}$ for all $a \in A$;

- $\left(\nabla_{v(a)} f\right)(a) \rightarrow 0$ as $a \rightarrow \infty$.

Such a $v$ exists by continuity and vanishing variation of $f$. Further, for each $a \in A$, let

$$
U_{a}=B_{X}(a, \sqrt{v(a)}),
$$

the open ball in $X$ about $a$ of radius $\sqrt{v(a)}$. Let $\rho: \mathbb{R}_{+} \rightarrow \mathbb{R}_{+}$be any non-decreasing, subadditive function such that $\rho(0)=0, \rho(t)>0$ for all $t>0, \rho(t) \rightarrow \infty$ as $t \rightarrow \infty$ and such that

$$
d(x, A) \leq \rho\left(d\left(x, a_{0}\right)\right) \text { implies } x \in \bigcup_{a \in A} U_{a}
$$

for all $x \in X$. One may then define

$$
F=\left\{x \in X \mid d(x, A) \leq \rho\left(d\left(x, a_{0}\right)\right)\right\},
$$

a closed subset of $X$ containing $A$ and covered by $\left\{U_{a}\right\}_{a \in A}$. Let $\left\{\phi_{i}\right\}_{i \in I}$ be a locally finite continuous partition of unity on $F$ subordinate to $\left\{U_{a}\right\}$. For each $i \in I$, let 
$a_{i} \in A$ be such that $\phi_{i}$ is supported in $U_{a_{i}}$. Using Tietze's extension theorem, extend each $\phi_{i}$ to a $[0,1]$-valued function defined on all of $X$ and supported in $U_{a_{i}}$. Finally, define a function

$$
\psi: X \rightarrow \mathbb{R}_{+}, \quad x \mapsto \max \left\{\frac{\rho\left(d\left(x, a_{0}\right)\right)-d(x, A)}{\rho\left(d\left(x, a_{0}\right)\right)}, 0\right\},
$$

(which is supported in $F$, and equal to one on $A$ ) and define

$$
\tilde{f}: X \rightarrow \mathbb{C}, \quad x \mapsto \psi(x) \sum_{i \in I} \phi_{i}(x) f\left(a_{i}\right)
$$

The choice of the cover $\left\{U_{a}\right\}$ guarantees that $\|f(a)-\tilde{f}(a)\|<\varepsilon$ for all $a \in A$, and moreover that $\tilde{f}$ is bounded, continuous, supported in $F$ and satisfies $f(x)-f(y) \in$ $\mathcal{K}$ for all $x, y \in X$. To finish the proof of the lemma it thus suffices to prove that $\tilde{f}$ is of vanishing variation.

To this end, say $x, y \in X$ satisfy $d(x, y) \leq R$ for some $R>0$. Then

$$
\begin{aligned}
& \|\tilde{f}(x)-\tilde{f}(y)\| \\
& \quad \leq|\psi(x)|\left\|\sum_{i \in I}\left(\phi_{i}(x)-\phi_{i}(y)\right) f\left(a_{i}\right)\right\|+|(\psi(x)-\psi(y))|\left\|\sum_{i \in I} \phi_{i}(y) f\left(a_{i}\right)\right\| \\
& \quad \leq|\psi(x)|\left\|\sum_{i \in I}\left(\phi_{i}(x)-\phi_{i}(y)\right) f\left(a_{i}\right)\right\|+|\psi(x)-\psi(y)|\|f\|_{\bar{c}^{\mathrm{red}}(A)} .
\end{aligned}
$$

Assume without loss of generality that $\psi(x), \psi(y)$ do not both vanish.

Case 1: Exactly one of $\psi(x), \psi(y)$ is zero.

Without loss of generality, assume that $\psi(x)$ vanishes. Then the above becomes

$$
\begin{aligned}
\|\tilde{f}(x)-\tilde{f}(y)\| & \leq|\psi(y)|\|f\| \\
& =\left(\frac{\rho\left(d\left(y, a_{0}\right)\right)-d(y, A)}{\rho\left(d\left(y, a_{0}\right)\right)}\right)\|f\| \\
& \leq\left(\frac{\rho\left(d\left(x, a_{0}\right)\right)+\rho(R)-d(x, A)+R}{\rho\left(d\left(y, a_{0}\right)\right)}\right)\|f\|
\end{aligned}
$$

using subadditivity of $\rho$. By assumption that $\psi(x)=0$, this in turn is bounded by

$$
\left(\frac{\rho(R)+R}{\rho\left(d\left(y, a_{0}\right)\right)}\right)\|f\|
$$

which gets arbitrarily small as long as $y$ gets far from $a_{0}$.

Case 2: Both of $\psi(x), \psi(y)$ are non-zero.

Using a similar argument to that in case $1,|\psi(x)-\psi(y)|$ can be made arbitrarily small by assuming that $x, y$ are suitably far from $a_{0}$. It thus remains to show that the term

$$
|\psi(x)|\left\|\sum_{i \in I}\left(\phi_{i}(x)-\phi_{i}(y)\right) f\left(a_{i}\right)\right\| \leq\left\|\sum_{i \in I}\left(\phi_{i}(x)-\phi_{i}(y)\right) f\left(a_{i}\right)\right\|
$$


can be made arbitrarily small by assuming $x$ and $y$ are suitably far from $a_{0}$. Now, by assumption that $\psi(x) \neq 0 \neq \psi(y)$, it follows that $x, y \in F$, whence there exist $a_{x}, a_{y} \in A$ such that $d\left(a_{x}, x\right) \leq \sqrt{v\left(a_{x}\right)}$ and $d\left(a_{y}, y\right) \leq \sqrt{v\left(a_{y}\right)}$. Moreover, as $v(a) \rightarrow \infty$ as $a \rightarrow \infty$ in $A$, if $x, y \in X$ are suitably far from $a_{0}$, then if $i \in I$ satisfies either $\phi_{i}(x) \neq 0$ or $\phi_{i}(y) \neq 0$, then $d\left(a_{i}, a_{x}\right) \leq v\left(a_{x}\right)$. Finally, then, by assumption that $\left(\nabla_{v(a)} f\right)(a) \rightarrow 0$ as $a \rightarrow \infty$,

$\left\|\sum_{i \in I}\left(\phi_{i}(x)-\phi_{i}(y)\right) f\left(a_{i}\right)\right\| \leq\left\|f\left(a_{x}\right)-\sum_{i \in I} \phi_{i}(x) f\left(a_{i}\right)\right\|+\left\|f\left(a_{x}\right)-\sum_{i \in I} \phi_{i}(y) f\left(a_{i}\right)\right\|$

can be made arbitrarily small for $a_{x}$ suitably far from $a_{0}$ (which in turn can be forced by stipulating that $x, y$ are suitably far from $a_{0}$ ). This completes the proof.

The existence of Mayer-Vietoris sequences as set out below follows from the two preceding lemmas.

Proposition 3.5. Let $X=A \cup B$ be a coarsely excisive decomposition and $i_{A}, i_{B}$, $j_{A}, j_{B}$ be as in Lemma 3.3 .

Then there exists a Mayer-Vietoris sequence

$$
\rightarrow K_{i}\left(\mathrm{c}^{\mathrm{red}}(X)\right) \stackrel{i_{A}^{*} \oplus i_{B}^{*}}{\longrightarrow} K_{i}\left(\mathrm{c}^{\mathrm{red}}(A)\right) \oplus K_{i}\left(\mathrm{c}^{\mathrm{red}}(B)\right) \stackrel{j_{A}^{*}-j_{B}^{*}}{\longrightarrow} K_{i}\left(\mathrm{c}^{\mathrm{red}}(A \cap B)\right) \rightarrow .
$$

It is moreover natural for morphisms of excisive decompositions in the category $\mathcal{C}$ in the obvious sense.

Proof. This is a special case of the general notion of Mayer-Vietoris sequence associated to a pullback diagram of $\mathrm{C}^{*}$-algebras (see for example [1], 21.3.2) such that the right-hand and bottom maps are surjective. The naturalness follows from naturalness for this general Mayer-Vietoris sequence with respect to morphisms of pullback diagrams.

This result seems to be of interest in itself; in what follows, however, we will only use a very special case of it as a crutch to Proposition 3.10.

The second ingredient needed for this proposition is a vanishing theorem for coarsely flasque spaces (cf. [10], Lemma 6.4.2). These are defined below.

Definition 3.6. $X$ is coarsely flasque if there exists a continuous coarse map $\phi: X \rightarrow$ $X$ such that:

- $\phi$ is close to the identity in the sense of Definition 2.1;

- for any compact subset $K \subseteq X$ there exists $N_{K} \in \mathbb{N}$ such that $\phi^{n}(x) \notin K$ for all $n \geq N_{K}$ and $x \in X$;

- for all $R>0$ there exists $S>0$ such that $d\left(\phi^{n}(x), \phi^{n}(y)\right) \leq S$ for all $x, y \in X$ with $d(x, y) \leq R$ and $n \in \mathbb{N}$. 
The prototypes of coarsely flasque spaces are those of the form $X=Y \times \mathbb{N}$ equipped with some natural product metric and with $\phi$ defined by $\phi:(y, n) \mapsto$ $(y, n+1)$. The examples we will actually use (in the proof of Proposition 3.10) are only slightly more complicated than this.

The following now gives a vanishing result for the K-theory of the corona functor. The proof is an Eilenberg swindle; as it is essentially the same as that of [4], Theorem 5.2, we only give a brief sketch.

Proposition 3.7. Let $X$ be a coarsely flasque space. Then

$$
K_{*}\left(c^{\mathrm{red}}(X)\right)=0
$$

Sketch of proof. Concretely realize $c^{\text {red }}(X)$ as maps into some $\mathcal{B}(\mathscr{H})$. Choose a decomposition $\mathscr{H}=\bigoplus_{n \in \mathbb{N}} \mathscr{H}_{n}$ of $\mathscr{H}$ into countably many infinite-dimensional subspaces and unitary isomorphisms $V_{n}: \mathscr{H} \rightarrow \mathscr{H}_{n}$. Define moreover conjugating maps

$$
\nu_{n}: T \mapsto V_{n} T V_{n}^{*}
$$

and (provisionally) define a $*$-homomorphism from $\bar{c}^{\text {red }}(X)$ to itself via the formula

$$
(v f)(x)=\bigoplus_{n \in \mathbb{N}} v_{n}\left(f \circ \phi^{n}(x)\right) .
$$

One needs to check that this is well defined and descends to a $*$-homomorphism from $c^{\text {red }}(X)$ to itself. Having done so, $v$ passes to an endomorphism $v_{*}$ of $K_{*}\left(c^{\text {red }}(X)\right)$. Note finally that if $x$ is any class in $K_{*}\left(c^{\text {red }}(X)\right)$ then $v_{*}(x)=$ $x+v_{*}(x)$, whence $x=0$ as required.

Example 3.8. We now have enough information to compute K-theory groups in the case of $\mathbb{R}^{n}$. Indeed, using Remark 2.4, part (2), one has that

$$
K_{i}\left(\mathrm{c}^{\mathrm{red}}(\text { point })\right)= \begin{cases}\mathbb{Z}, & i=1, \\ 0, & i=0 .\end{cases}
$$

Using this, and an application of the Mayer-Vietoris sequence from Proposition 3.5 to the coarsely excisive decomposition $\mathbb{R}=(-\infty, 0] \cup[0, \infty)$ from Example 3.2, and using also Proposition 3.7 to show that $K_{*}\left(c^{\text {red }}((-\infty, 0])\right)=K_{*}\left(c^{\text {red }}([0, \infty))\right)=0$, one gets that

$$
K_{i}\left(\mathrm{c}^{\mathrm{red}}(\mathbb{R})\right)= \begin{cases}\mathbb{Z}, & i=0, \\ 0, & i=1\end{cases}
$$

The general case now follows from the coarsely excisive decomposition $\mathbb{R}^{n}=\mathbb{R}^{n-1} \times$ $(-\infty, 0] \cup \mathbb{R}^{n-1} \times[0, \infty)$, induction and Propositions 3.7 and 3.5 again; one sees that

$$
K_{i}\left(\mathrm{c}^{\mathrm{red}}\left(\mathbb{R}^{n}\right)\right)= \begin{cases}\mathbb{Z}, & i=n+1 \bmod 2, \\ 0, & i=n \bmod 2 .\end{cases}
$$


See for example [10], Theorem 6.4.10, for the corresponding computation in the case of the Roe algebra.

The results we have so far can now be put together to yield a homotopy invariance result; the notion of homotopy we use is almost the same as that from [7], Section 11.

Definition 3.9. Let $\phi_{0}, \phi_{1}: X \rightarrow Y$ be morphisms in the category $\mathcal{C}$. They are said to be elementarily coarsely homotopic if there exists a map $H: X \times \mathbb{N} \rightarrow Y$ and a coarse map $X \rightarrow \mathbb{N}$ denoted $x \mapsto n_{x}$ such that:

- $H(x, 0)=\phi_{0}(x)$ for all $x \in X$;

- for all $x \in X$ and all $n \geq n_{x}, H(x, n)=\phi_{1}(x)$;

- if $Z:=\left\{(x, n) \in X \times \mathbb{N} \mid n \leq n_{x}\right\}$ is equipped with the restriction of the metric $d((x, n),(y, m)):=d_{X}(x, y)+|n-m|$ from $X \times \mathbb{N}$, then $H$ restricted to $Z$ is a coarse map.

Such an $H$ is called an elementary coarse homotopy between $\phi_{0}$ and $\phi_{1}$.

Coarse homotopy is then the equivalence relation on morphisms in $\mathcal{C}$ generated by elementary coarse homotopy.

As an example, note that while the Euclidean and hyperbolic planes are not isomorphic in the category $\mathcal{C}$, they are 'coarse homotopy equivalent': the inverse of the exponential map, which goes from the manifold to its tangent space at a fixed basepoint, is a coarse homotopy equivalence.

The proof of the following theorem is precisely the same as that of [10], Proposition 12.4.12; indeed, one can interpret that theorem as showing that any functor from $\mathcal{C}$ to abelian groups with Mayer-Vietoris sequences and that vanishes on flasque spaces is coarse homotopy invariant. For the reader's convenience we give a brief sketch.

Proposition 3.10. Let $\phi_{0}, \phi_{1}: X \rightarrow Y$ be morphisms in the category $C$ that are coarsely homotopic. Then the induced maps

$$
\phi_{0}^{*}, \phi_{1}^{*}: K_{*}\left(\mathrm{c}^{\mathrm{red}}(Y)\right) \rightarrow K_{*}\left(\mathrm{c}^{\mathrm{red}}(X)\right)
$$

are the same.

Sketch of proof. It suffices to show the result when $\phi_{0}, \phi_{1}$ are elementarily coarsely homotopic. Let $H: X \times \mathbb{N} \rightarrow Y$ be an elementary coarse homotopy between them, which we extend to all of $X \times \mathbb{Z}$ by defining $H(x, n)=H(x, 0)$ for $n<0$. Let

$$
Z:=\left\{(x, n) \in X \times \mathbb{Z} \mid x \in X, 0 \leq n \leq n_{x}\right\}
$$

be as in the definition of elementary coarse homotopy and define inclusion maps $i_{0}, i_{1}: X \rightarrow Z$ by

$$
i_{0}(x)=(x, 0) \quad \text { and } \quad i_{1}(x)=\left(x, n_{x}\right)
$$


$i_{0}$ is clearly coarse, while $i_{1}$ is coarse by definition of an elementary coarse homotopy. Note also that $H$ is coarse when restricted to $Z$, and that

$$
H \circ i_{j}=\phi_{j} \quad \text { for } j=0,1 ;
$$

it thus suffices to show that the maps

$$
i_{0}^{*}, i_{1}^{*}: K_{*}\left(c^{\mathrm{red}}(X) \rightarrow K_{*}\left(\mathrm{c}^{\mathrm{red}}(Z)\right)\right.
$$

are in fact equal.

Let $\pi$ be the coordinate projection from $X \times \mathbb{Z}$ to $X$. Let $A=\{(x, n) \in X \times \mathbb{Z} \mid$ $n \leq 0\}, A^{\prime}=\left\{(x, n) \in X \times \mathbb{Z} \mid n \leq n_{x}\right\}$ and $B=\{(x, n) \in X \times \mathbb{Z} \mid n \geq 0\}$. Then both of the decompositions $X \times \mathbb{Z}=A \cup B$ and $X \times \mathbb{Z}=A^{\prime} \cup B$ are coarsely excisive; a commutative diagram of the associated Mayer-Vietoris sequences together with coarse flasqueness of $A, A^{\prime}, B$ then shows that $i_{0}^{*}: K_{*}\left(c^{\text {red }}(Z)\right) \rightarrow K_{*}\left(c^{\text {red }}(X)\right)$ is an isomorphism.

As $\pi^{*}$ is a one-sided inverse to both $i_{0}^{*}$ and $i_{1}^{*}$, it now follows that all three of these maps are isomorphisms, and moreover that $i_{0}^{*}, i_{1}^{*}$ are both equal to $\left(\pi^{*}\right)^{-1}$.

For the last two results of this section, we return to the stable Higson compactification. Firstly, define a notion of continuous coarse homotopy by forcing all maps in Definition 3.9 to be continuous, and replacing $\mathbb{N}$ with $\mathbb{R}_{+}$. Using essentially the same ingredients as in Proposition 3.10 (see [15], Chapter 4, for details), we then have the following homotopy invariance result.

Proposition 3.11. Let $\phi_{0}, \phi_{1}: X \rightarrow Y$ be morphisms in the category ee that are continuously coarsely homotopic. Then the induced maps

$$
\phi_{0}^{*}, \phi_{1}^{*}: K_{*}\left(\bar{c}^{\mathrm{red}}(Y)\right) \rightarrow K_{*}\left(\bar{c}^{\mathrm{red}}(X)\right)
$$

are the same.

The last result in this section is another vanishing result, closely related to [4], Proposition 8.9.

Definition 3.12. Let $X$ be a proper metric space. A continuous coarse map $s: X \rightarrow X$ is called a scaling if $d(s(x), s(y)) \leq d(x, y) / 2$ for all $x, y \in X$.

Proposition 3.13. Say $s: X \rightarrow X$ is a scaling. Then the map

$$
s^{*}: K_{*}\left(\bar{c}^{\mathrm{red}}(X)\right) \rightarrow K_{*}\left(\overline{\mathrm{c}}^{\mathrm{red}}(X)\right)
$$

is zero.

The idea of the proof is essentially the same as that of [10], Theorem 12.4.11, so we only give a sketch below. For details see [15], Proposition 4.3.16. 
Sketch of proof. Choose a decomposition $\mathscr{H}=\bigoplus_{n \in \mathbb{N}} \mathscr{H}_{n}$, and corresponding unitaries $V_{n}$ and conjugating maps $v_{n}$ as in Proposition 3.7. Define (provisionally) a map from $\bar{c}^{\text {red }}(X)$ to itself via the formula

$$
(v f)(x)=\bigoplus_{n \in \mathbb{N}} v_{n}\left(f \circ s^{n}(x)\right) ;
$$

note that $v$ does not preserve $C_{0}(X, \mathcal{K})$, so $v$ does not pass to the corona $c^{\text {red }}(X)$.

To show that $v$ is well defined, we must check that for any $f \in \bar{c}^{\text {red }}(X), v f$ is continuous, of vanishing variation, and such that $v f(x)-v f(y) \in \mathcal{K}$ for all $x, y \in$ $X$. These all follow readily once we have observed that because $f$ is of vanishing variation it is uniformly continuous; hence the (global) modulus of continuity of $f \circ s^{n}$ gets arbitrarily small as $n$ tends to infinity.

The proof is now completed by an Eilenberg swindle just as in the case of the proof of Proposition 3.7.

\section{Some examples}

In this section we will use the properties above to compute some examples. These all rely on the following definition.

Definition 4.1. A geometric compactification of $X$ is a compactification $\bar{X}$ such that any continuous function $f: \bar{X} \rightarrow \mathbb{C}$ is of vanishing variation when restricted to $X$.

A geometric boundary of $X$ is a corona $\partial X:=\bar{X} \backslash X$ associated to a geometric compactification of $X$.

Examples 4.2. The two most interesting examples for us both come from notions of non-positive curvature. For background and precise definitions in both cases, see [2].

(1) Say $X$ is a proper geodesic CAT(0) metric space. Then the visual compactification $\bar{X}^{v}$, and associated visual boundary $\partial_{v} X$, of $X$ are geometric. For example, $X$ might be a simply connected manifold of non-positive sectional curvature, in which case $\partial_{v} X$ is a (topological) sphere. A different example is given when $X$ is an affine Bruhat-Tits building, in which case $\partial_{v} X$ is homeomorphic to the Borel-Serre boundary of $X$.

(2) Say $X$ is a Gromov hyperbolic space. Then the Gromov boundary $\partial_{G} X$ is a geometric boundary of $X$. The main examples of interest here are perhaps word hyperbolic groups and simply connected manifolds of strictly negative curvature.

These examples are well known to experts in the area, so we will not give a proof here; see [15], Lemma 4.6.2, for a proof in the CAT(0) case, and [14], Proposition 2.3, for a proof in the Gromov hyperbolic case.

Now, if $\partial X$ is a geometric boundary of $X$ then there is a canonical inclusion

$$
i: C(\partial X) \otimes \mathcal{K} \rightarrow \mathfrak{c}(X)
$$


(cf. [4], Proposition 3.6). In the case of the examples in 4.2 above, we can use the results of Section 3 to show that these maps induce isomorphisms on K-theory. In both cases, we only sketch the proofs here; complete proofs can be found in [15], Section 24.6 and 4.7 .

Proposition 4.3. Say that $X$ is a proper geodesic CAT(0) metric space. Then the inclusion

$$
i: C\left(\partial_{v} X\right) \otimes \mathcal{K} \rightarrow c(X)
$$

from (4) above induces an isomorphism on K-theory.

Sketch of proof. Fix a basepoint $x_{0} \in X$. Then for any $x \in X$, there is a unique geodesic segment $\gamma_{x}$ from $x_{0}$ to $x$. Define by $s(x)=\gamma_{x}\left(d\left(x_{0}, x\right) / 2\right)$ a map $s: X \rightarrow X$; it follows from the $\mathrm{CAT}(0)$ inequality that $s$ is a scaling. It is moreover continuously coarsely homotopic to the identity via the homotopy

$$
H: X \times \mathbb{R}_{+} \rightarrow X, \quad(x, t) \mapsto \begin{cases}\gamma_{x}\left(d\left(x, x_{0}\right) / 2+t\right), & 0 \leq t \leq d\left(x, x_{0}\right) / 2, \\ x, & t>d\left(x, x_{0}\right) / 2 .\end{cases}
$$

Hence by Propositions 3.11 and 3.13, the map $s^{*}: K_{*}\left(\bar{c}^{\mathrm{red}}(X)\right) \rightarrow K_{*}\left(\bar{c}^{\mathrm{red}}(X)\right)$ induced by $s$ is both zero and the identity. It follows from Proposition 2.3 that $K_{*}\left(\bar{c}^{\text {red }}(X)\right)$ is zero, whence $K_{*}(\bar{c}(X)) \cong \mathbb{Z}$, with a generator being given by any constant map to a rank one projection.

Finally, consider the commutative diagram

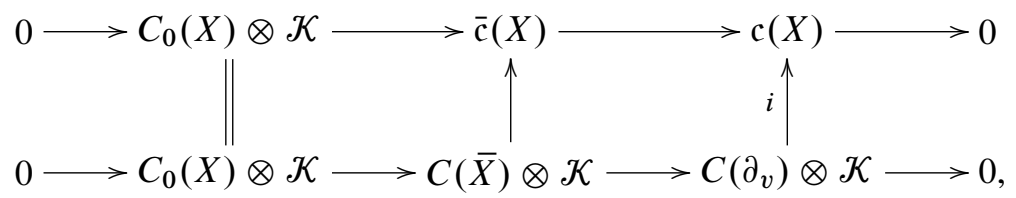

where the vertical maps are induced by inclusion. As the visual compactification is contractible, the argument above shows that the central vertical map induces an isomorphism on $\mathrm{K}$-theory. Hence $i$ induces an isomorphism on $\mathrm{K}$-theory by the five lemma.

Note that in some cases of interest, for example manifolds of non-positive curvature, the proposition allows for very explicit descriptions of the generators of $K_{*}\left(\mathrm{c}^{\text {red }}(X)\right)$ in terms of Bott-type elements. The following example also helps make the isomorphism induced by $i$ a little more explicit in some sense.

Example 4.4. If $X$ is as in the proposition above, one can also construct an explicit inverse to $i_{*}$ using asymptotic morphisms. Indeed, fix a basepoint $x_{0}$ in $X$, and for each $t>0$, let $S_{t}$ denote the sphere of radius $t$ about $x_{0}$. For each $t \geq s$, there are natural (and compatible) projections $p_{t, s}: S_{t} \rightarrow S_{s}$ such that $\partial_{v} X$ can be defined 
as the limit of the inverse system $\left(S_{t}, p_{t, s}\right)$ (see [2], II.8.5, for details). Hence in particular for each $t>0$, there is a natural inclusion map $i_{t}: C\left(S_{t}\right) \rightarrow C\left(\partial_{v} X\right)$.

Define now

$$
e_{t}: \bar{c}(X) \rightarrow C\left(\partial_{v} X\right) \otimes \mathcal{K}
$$

to be the composition of restriction to $S_{t}$ and (the stabilization of) $i_{t}$. Taking quotients by functions vanishing at infinity on both sides, the maps $e_{t}$ fit together to give a $*$ homomorphism

$$
\alpha: c(X) \rightarrow \frac{C_{b}\left([0, \infty), C\left(\partial_{v} X\right) \otimes \mathcal{K}\right)}{C_{0}\left([0, \infty), C\left(\partial_{v} X\right) \otimes \mathcal{K}\right)}
$$

(this is not true before passing to quotients - consider a three-regular tree), or in other words an asymptotic morphism from $\mathrm{c}(X)$ to $C\left(\partial_{v} X\right) \otimes \mathcal{K}$.

It is not hard to see that if $\alpha_{*}: K_{*}(\mathfrak{c}(X)) \rightarrow K_{*}\left(C\left(\partial_{v} X\right) \otimes \mathcal{K}\right)$ is the map on $\mathrm{K}$-theory induced by $\alpha$, then $\alpha_{*} \circ i_{*}$ is the identity on $K_{*}\left(C\left(\partial_{v} X\right) \otimes \mathcal{K}\right)$. As moreover $i_{*}$ is an isomorphism, one must have that $\alpha_{*}$ is in fact a two-sided inverse to $i_{*}$; I do not, however, know how to prove this latter fact directly. Having this explicit inverse to $i_{*}$ is important for the index theorem of [16].

Proposition 4.5. Say that $X$ is a proper quasi-geodesic Gromov hyperbolic space. Then the inclusion

$$
i: C\left(\partial_{G} X\right) \otimes \mathcal{K} \rightarrow c(X) .
$$

from (4) above induces an isomorphism on K-theory.

Sketch of proof. Embed the Gromov boundary $\partial_{G} X$ as a subset of the unit sphere of some Euclidean space $\mathbb{R}^{n}$ (as $\partial_{G} X$ is compact and finite dimensional, such an embedding always exists). Define the open cone over $\partial_{G} X$ to be

$$
\mathcal{O} \partial_{G} X:=\left\{t x \in \mathbb{R}^{n} \mid t \in \mathbb{R}_{+}, x \in \partial_{G} X\right\} .
$$

Note that this admits a (geometric) compactification by $\partial_{G} X$, and essentially the same argument as used in the proof of the previous proposition shows that the inclusion

$$
i^{\mathcal{O}}: C\left(\partial_{G} X\right) \otimes \mathcal{K} \rightarrow \mathfrak{c}\left(\mathcal{O} \partial_{G} X\right)
$$

induces an isomorphism on K-theory. On the other hand, one can argue as in [9] that there is an 'exponential map' exp: $\mathcal{O} \partial_{g} X \rightarrow X$; roughly, points of $\partial_{G} X$ are given as equivalence classes $[\gamma]$ of quasi-geodesic rays in $X$, and the map exp takes a point $t[\gamma] \in \mathcal{O}\left(\partial_{G} X\right)$ to $\gamma(t)$. One can then use our homotopy invariance results to show that (a slightly altered version of) exp induces an isomorphism

$$
\exp ^{*}: K_{*}(\mathfrak{c}(X)) \rightarrow K_{*}\left(\mathfrak{c}\left(\mathcal{O} \partial_{G} X\right)\right)
$$

(for details of this argument, again see [15], Section 4.7). We now have that the composition

$$
\left(\exp ^{*}\right)^{-1} \circ i_{*}^{\mathcal{O}}: K_{*}\left(C\left(\partial_{G} X\right) \otimes \mathcal{K}\right) \rightarrow K_{*}(\mathrm{c}(X))
$$


is an isomorphism; a computation showing that it is equal to $i_{*}$ now completes the proof.

To conclude this piece, we have the following application to the coarse coassembly map and strong Novikov conjecture.

Corollary 4.6. Let $X$ be a space as in either of Propositions 4.3 or 4.5 above, and assume moreover that $X$ is uniformly contractible. Then the coarse co-assembly map for $X$ is an isomorphism.

As a corollary, the strong Novikov conjecture holds for any torsion-free group with finite classifying space acting properly cocompactly by isometries on a proper geodesic CAT(0) space, or for any torsion-free Gromov hyperbolic group.

Proof. Let $X$ be as in the statement of the theorem, let $\partial X$ denote the relevant geometric boundary (either the visual boundary, or Gromov boundary as appropriate), and $\bar{X}$ the associated compactification. There is then a commutative diagram

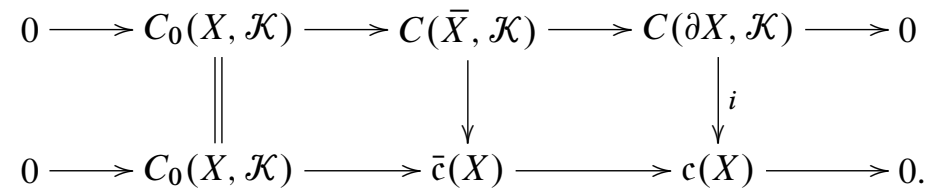

As the left- and right-hand vertical maps induce isomorphisms on K-theory, the central one must too; moreover, as $\bar{X}$ is contractible (see [15], Lemma 4.6.1), this implies that the inclusion $\mathcal{K} \rightarrow \overline{\mathfrak{c}}(X)$ as constant functions induces an isomorphism on $\mathrm{K}$ theory. From Proposition 2.3, part 2, then, $K_{*}\left(\bar{c}^{\text {red }}(X)\right)=0$. However, in the case of uniformly contractible $X$, the coarse co-assembly is defined to be the $\mathrm{K}$-theory boundary map associated to the short exact sequence

$$
0 \rightarrow C_{0}(X, \mathcal{K}) \rightarrow \bar{c}^{\mathrm{red}}(X) \rightarrow \mathrm{c}^{\mathrm{red}}(X) \rightarrow 0
$$

and the fact that is an isomorphism follows from the K-theory long exact sequence.

The statement for a group acting properly cocompactly by isometries on a proper CAT(0) space $X$ follows from [5], Theorem 5, the fact that a group satisfying the hypotheses given is coarsely equivalent to the space $X$, the fact that the coarse coassembly map is invariant under coarse equivalences, and the fact that a contractible space admitting a cocompact group action is automatically uniformly contractible. The statement for a Gromov hyperbolic group $\Gamma$ follows by letting $X$ be its Rips complex $P_{d}(\Gamma)$ for some $d$ suitably large (see [6], Chapter 4, and the same argument as in the $\mathrm{CAT}(0)$ case.

Acknowledgments. I would like to thank my advisor John Roe for his support, encouragement and many helpful comments during work on this piece. 


\section{References}

[1] B. Blackadar, K-theory for operator algebras. 2nd ed., Math. Sci. Res. Inst. Publ. 5, Cambridge University Press, Cambridge 1998. Zbl 0913.46054 MR 1656031

[2] Metric spaces of non-positive curvature. Grundlehren Math. Wiss. 319, Springer-Verlag, Berlin 1999. Zbl 0988.53001 MR 1744486

[3] A. N. Dranishnikov, J. Keesling, and V. V. Uspenskij, On the Higson corona of uniformly contractible spaces. Topology 37 (1998), 791-803. Zbl 0910.54026 MR 1607744 (99k:57049)

[4] H. Emerson and R. Meyer, Dualizing the coarse assembly map. J. Inst. Math. Jussieu 5 (2006), 161-186. Zbl 1092.19005 MR 2225040

[5] H. Emerson and R. Meyer, A descent principle for the Dirac-dual-Dirac method. Topology 46 (2007), 185-209. Zbl 1119.19005 MR 2313071

[6] É. Ghys and P. de la Harpe (eds.), Sur les groupes hyperboliques d'après Mikhael Gromov. Progr. Math. 83, Birkhäuser, Boston 1990. Zbl 0731.20025 MR 1086648

[7] N. Higson, E. K. Pedersen, and J. Roe, $C^{*}$-algebras and controlled topology. K-Theory 11 (1997), 209-239. Zbl 0879.19003 MR 1451755

[8] N. Higson and J. Roe, A homotopy invariance theorem in coarse cohomology and $K$ theory. Trans. Amer. Math. Soc. 345 (1994), 347-365. Zbl 0812.58083 MR 1243611

[9] N. Higson and J. Roe, On the coarse Baum-Connes conjecture. In Novikov conjectures, index theorems and rigidity, Vol. 2 (Oberwolfach, 1993), London Math. Soc. Lecture Note Ser. 227, Cambridge University Press, Cambridge 1995, 227-254. Zbl 0957.58019 MR 1388312

[10] N. Higson and J. Roe, Analytic K-homology. Oxford Math. Monogr., Oxford University Press, Oxford 2000. Zbl 0968.46058 MR 1817560

[11] N. Higson, J. Roe, and G. L. Yu, A coarse Mayer-Vietoris principle. Math. Proc. Cambridge Philos. Soc. 114 (1993), 85-97. Zbl 0792.55001 MR 1219916

[12] V. S. Rabinovich, S. Roch, and J. Roe, Fredholm indices of band-dominated operators. Integral Equations Operator Theory 49 (2004), 221-238. Zbl 1068.47016 MR 2060373

[13] V. Rabinovich, S. Roch, and B. Silbermann, Limit operators and their applications in operator theory. Oper. Theory Adv. Appl. 150, Birkhäuser, Basel 2004. Zbl 1077.47002 MR 2075882

[14] J. Roe, Hyperbolic metric spaces and the exotic cohomology Novikov conjecture. $K$ Theory 4 (1990/91), 501-512. Zbl 0756.55003 MR 1123175

[15] R. Willett, Band-dominated operators and the stable Higson corona. Ph.D. thesis, The Pennsylvania State University, 2009; Proquest, Umi Dissertation Publishing, Ann Arbor, MI, 2011.

[16] R. Willett, An index theorem for band-dominated operators with slowly oscillating coefficients (after Deundyak and Shteinberg). Integral Equations Operator Theory 69 (2011), 301-316. Zbl 05898356 MR 2774605

Received December 1, 2010; revised December 15, 2010 
R. Willett, Department of Mathematics, University of Hawaii at Manoa, 2565 McCarthy Mall, Honolulu, Hawaii 96822, U.S.A.

E-mail: rufus.willett@hawaii.edu 